ARTÍCULO PRODUCTO DE INVESTIGACIÓN

\title{
Condiciones para el rescate, fomento y preservación de una lengua nativa en educación básica
}

Conditions for the rescue, promotion and preservation of a native language in basic education

\author{
Magda Gabriela Sánchez Trujillo ${ }^{1}$ \\ Universidad Autónoma del Estado de Hidalgo \\ Jessica Hernández Ángeles ${ }^{2}$ \\ Escuela Primaria Bilingüe Francisco I Madero
}

Recibido: 11.12 .2020

Aceptado: 26.12.2020

\section{Resumen}

El objetivo del presente estudio es identificar cuáles son las condiciones que facilitan el rescate, fomento y preservación de la lengua Hñahñu en la Escuela Primaria Bilingüe Francisco I. Madero, ubicada en una comunidad en el Valle del Mezquital en el Estado de Hidalgo, México. Se toma como marco de referencia el Plan y los Programas de Estudio de la Educación Básica 2011 que perfila el aprendizaje de las lenguas indígenas reconocidas en México. Metodológicamente se realizó una investigación exploratoria con enfoque cualitativo. Los resultados indican que tanto docentes como alumnos tienen disposición para rescatar la lengua, ya que la consideran parte de su cultura ancestral.

\footnotetext{
${ }^{1}$ magdags@uaeh.edu.mx http://orcid.org/0000-0001-6446-0901

2 jesi_dj@hotmail.fr
} 
Palabras clave: Lengua nativa, Hñahñu, Aprendizaje.

\begin{abstract}
The objective of this study is to identify the conditions that facilitate the rescue, promotion and preservation of the Hñahñu language in the Francisco I. Madero Bilingual Primary School, a community located in the Valle del Mezquital in the State of Hidalgo, Mexico. Is taken as a reference the Plan and Programs Study 2016 Basic Education outlining learning of indigenous languages recognized in Mexico. Methodologically an exploratory research was conducted with qualitative approach. The results indicate that both teachers and students are willing to rescue the language, since they consider it part of their ancestral culture.
\end{abstract}

Keywords: native language, Hñahñu, learning.

México, es una de las 10 naciones con mayor diversidad lingüística del mundo: 7 millones de mexicanos hablan una de las 68 lenguas originarias, sin contar las 364 variantes lingüísticas o dialectos. De ellas, la tercera parte se encuentra en alto riesgo de desaparecer UNESCO (2003).

Con la premisa anterior, el país está conformado por zonas con diversidades lingüísticas las cuales se encuentran en riesgo de eliminarse o disminuir el número de hablantes. Es por esto, que la Ley de Derechos Lingüísticos de los pueblos Indígenas (2005) contiene disposiciones destinadas a promover revalorar y revitalizar las lenguas originales como la lengua Hñahñu.

Aunado a los cambios en la Constitución política de los estados unidos mexicanos aprobados por el congreso, se busca generar en el sistema educativo de nivel básico las condiciones para rescatar, fomentar y preservar la lengua nativa. A su vez, la reforma es compatible con el artículo 28 del convenio 169 sobre los pueblos indígenas y tribales de países independientes de la organización internacional del trabajo. 
A partir de este planteamiento, se encuentra que es escaso el material que apoya en el proceso de aprendizaje a los alumnos y maestros debido a lo poco lo que se ha hecho al respecto, de ahí la inquietud de diseñar y proponer estrategias que posibiliten construir conocimientos a partir de una comunicación social, que permita compartir e involucrar a la comunidad estudiantil, siendo la escritura, una parte esencial para lograrlo.

Para dar cuenta clara del planteamiento, el estudio se realizó en la escuela primaria bilingüe Francisco I. Madero de la comunidad de Huitexcalco de Morelos, municipio de Chilcuautla en el Estado de Hidalgo, esta escuela pertenece al subsistema de educación indígena, el cual tiene como tarea promover una comunicación bilingüe, a través de la práctica y conocimiento del español y de la lengua Hñahñu.

La población cuenta aproximadamente con 668 habitantes: 351 mujeres y 317 hombres, de los cuales 74 son analfabetas. Su nivel cultural se encuentra entre primaria y secundaria y existen pocos profesionistas. De la población hablante de la lengua Hñahñu 124 son hombres y 116 mujeres sumando un total de 345 habitantes mayores de 20 años, según el censo general de población recabado en el año 2016.

Aunque el nombre de la comunidad proviene del náhuatl, ésta es de origen Hñahñu. Una parte de la población adulta considerando a padres de familia, pero sobre todo los ancianos hablan la lengua indígena (bilingües, español-Hñahñu), poco la utilizan en conversaciones dentro de la comunidad o familia, por lo cual está desapareciendo; de ahí la importancia de impulsar la lecto-escritura de esta lengua.

Lo anterior, es bajo el supuesto de que la lengua Hñahñu es el dialecto materno de todos los niños que acuden a la institución por ende se rigen con los lineamientos de Educación Indígena, más aun, en los libros de texto se encuentran contenidos para la enseñanza de la lectura y escritura de la lengua autóctona de una forma homogénea, sin embargo; en la comunidad e institución de estudio la lengua ha sido desplazada por el español, al punto de que ahora ésta es la lengua materna de la mayoría de los alumnos. 
Para ello, es necesario mediante la escuela recuperar actividades dentro del aula que interesen y motiven la enseñanza y el aprendizaje de la lengua indígena, Díaz (1998) hace mención que el lenguaje es un sistema de comunicación, y las lenguas no son sino casos particulares del lenguaje humano. Desde esta cosmovisión, es de suma importancia para todos quienes están inmersos en educación indígena abordar con seriedad las estructuras gramaticales sin obstaculizar las fronteras lingüísticas.

La Ley General de Derechos Lingüísticos de los Pueblos Indígenas (2010), en el artículo 5 establece que a través de sus tres órdenes de gobierno, -Federación, Entidades Federativas y municipios-, en los ámbitos de sus respectivas competencias, reconocer, proteger y promover la preservación, desarrollo y uso de las lenguas indígenas nacionales, es la razón de porque las autoridades educativas hacen hincapié en que se lleve a cabo actividades para tal índole, con el propósito de cumplir con los objetivos y lineamientos establecidos en el programa de estudio de este nivel, en específico referente a la lecto-escritura de la lengua Hñahñu como medio para preservar su uso.

De ahí que, durante la enseñanza de la lengua indígena, se percibe que los niños conocen el significado de algunas palabras, así como el interés por aprender y conocer más, no obstante los docentes detectan problemas de escritura, por ejemplo escriben: satio (perro), oni (gallina) dambri (vaca) palabras, que de acuerdo a las normas ortográficas para la escritura de la lengua Hñahñu se deben escribir; st’atyo (perro), oni (gallina), ndämfri (vaca).

El Instituto Lingüístico de Verano (ILV, 2016) identifica que la familia otopame es una rama importante en el tronco otomangue que incluye varias lenguas en el centro de México. Tal es el caso del otomí (hñahñu) que forma parte de un grupo de seis variedades que se hablan en los estados de Puebla, Veracruz, Querétaro, Hidalgo y Tlaxcala.

En los últimos acuerdos de las normas ortográficas la lengua Hñahñu consta de 20 consonantes y 9 vocales para el alfabeto Hñahñu. 
$\mathrm{a}, \ddot{a}, \mathrm{~b}, \mathrm{~d}, \mathrm{e}, \underline{\mathrm{e}}, \mathrm{f}, \mathrm{g}, \mathrm{h}, \mathrm{i}, \mathrm{j}, \mathrm{k}, \mathrm{m}, \mathrm{n}, \tilde{\mathrm{n}}, \mathrm{o}, \underline{\mathrm{o}}, \mathrm{p}, \mathrm{r}, \mathrm{s}, \mathrm{t}, \mathrm{th}, \mathrm{ts}, \mathrm{u}, \underline{\mathrm{u}}, \mathrm{x}, \mathrm{y}, \mathrm{z}$, '.

Como se puede ver las consonantes tienen combinaciones que dificultan la pronunciación, al igual que las vocales $\ddot{a}, \underline{\mathrm{e}}, \underline{\mathrm{o}}, \underline{\mathrm{u}}$.

Así mismo, hay palabras que se escriben de acuerdo al sonido y en las que no existe mayor dificultad a la hora de escribirlas pues el sonido es similar, por ejemplo; mixi (gato), menja (gallo), fani (caballo).

En las oraciones, y de acuerdo a las normas ortográficas no se escribe como tal se pronuncia, en la traducción se invierten algunas palabras o se antepone Ra o Ya antes de un sustantivo.

Ejemplo.

\begin{tabular}{|l|l|l|}
\hline Enunciado. & Traducción del niño. & Lo correcto. \\
\hline María come tortillas calientes. & (María tsi hme pa) & Ra María tsi yä pahme \\
\hline El pan es sabroso. & (Ra thume xa kuhi) & Ra thuhme xa kuhi. \\
\hline
\end{tabular}

Ante el análisis y reflexión de la práctica docente en el proceso de enseñanza aprendizaje de la asignatura de Hñahñu, se detecta al momento, una omisión importante de normas ortográficas dificultando la escritura. Al no encontrar una respuesta favorable para enfrentar y solucionar esta situación, se busca diseñar estrategias que permitan entender la producción de textos en lengua indígena.

Es importante mencionar, el interés que muestran los padres de familia por lograr conocimientos referentes a la lengua autóctona ya que durante el desarrollo de las actividades con los alumnos resalta el apoyo que brindan a sus hijos sin importar que ellos son monolingües en español, pues consideran que la lengua es un elemento de identidad (Bonfil, 1990, p. 48) hace mención que la identidad es saberse y asumirse como integrante de un pueblo y ser reconocido por propios y extraños, formar parte de una sociedad con una propia cultura, esto motiva a los docentes para ahondar en su conocimiento. 
Este interés vivo y manifiesto por parte de alumnos y padres de familia, ha motivado al grupo de maestros que forman parte de la escuela primaria ir en busca de talleres, diplomados o cursos de Hñahñu con el propósito de conocer y practicar la lengua acorde al contexto del estudiante con contenidos propios de la región los cuales permitan abordar y mejorar este campo del conocimiento.

Con el propósito de proteger y fomentar la lengua, se rescata la oralidad y fomenta la escritura de la lengua indígena, ya que a través de ella los alumnos adquieren mayor valoración y sentido como parte de su cultura al tiempo de alcanzar cierto dominio de la lectura y escritura.

La educación indígena fue establecida para integrar a los pueblos indígenas a la sociedad nacional, es por ello que uno de los objetivos relevantes fue castellanizar las escuelas y con ello el español fuese utilizado para interactuar con el contexto nacional. Una especie de asesinato académico, buscando la castellanización de los pueblos naturales (Díaz, 2012, 67).

Al plantear la enseñanza de la lectura y escritura de la lengua indígena Hñahñu, se quiere retomar un tema que ha sido relevante por varios periodos escolares principalmente para docentes, directivos e instituciones e incluso para el subsistema de educación Indígena, pues éste ha sido creado, para dar respuesta a políticas nacionales.

Con estos antecedentes, se da cuenta del panorama que representa la enseñanza en educación indígena al propiciar que los alumnos se apropien de la lengua Hñahñu ya que, una de las normas establecidas actualmente en el acuerdo 592 es que el Plan y los Programas de Estudio de la Educación Básica 2016 deben favorecer un currículo que beneficie el aprendizaje de los alumnos en su lengua materna, sea ésta el español o alguna de las lenguas indígenas reconocidas en México.

Este enfoque, plantea el propósito de integrar a la población indígena al tomar en cuenta la interculturalidad, siendo la lengua un elemento característico, por tal razón surge la importancia de abordar la enseñanza de la lectura y escritura del dialecto autóctono por medio 
de escritos, los cuales se socializarán al compartir con sus familiares, amigos y padres, con el objetivo de rescatar la modalidad escrita.

Es decir, que la escuela es responsable de fomentar el rescate cultural y lingüístico de los grupos indígenas porque permitirá desarrollar la cultura, los conocimientos de cada etnia, tanto como conocer los valores científicos y culturales de otros pueblos, para ello es necesario reconocer que el lenguaje es una actividad humana que establece la interacción social lingüística en el desarrollo del lenguaje, ya que éste juega un papel importante para la comunicación del niño.

Por ello, es necesario alcanzar uno de los objetivos primordiales de la educación indígena que es garantizar que prevalezca la cultura por medio de registros escritos y con ello la importancia de que los adultos sean quienes transmitan conocimiento a las nuevas generaciones, quienes a su vez tendrán la responsabilidad de conservar, enriquecer, adquirir y transmitir todo el bagaje lingüístico.

Es decir, difundir de forma veraz la lengua entre maestros y alumnos que, al practicarla, sea funcional, logrando un bilingüismo eficaz, sin demerito de alguna de ellas, lo anterior se reitera con artículo 4 de la Ley General de Derechos Lingüísticos de los Pueblos Indígenas (2010) que hace referencia al reconocer que el español y las lenguas indígenas son lenguas nacionales por su origen histórico, y tienen la misma validez en su territorio, localización y contexto en que se hablen.

De esta manera, el propósito del presente estudio es identificar el nivel de alfabetización bilingüe, mediante la producción de textos desde la perspectiva del personal docente y alumnos de la institución primaria, con la finalidad de preservar, promover y rescatar la lengua Hñahañu. 


\section{Educación indígena}

Los Lineamientos generales de educación intercultural bilingüe para niños y niñas indígenas (2000), tienen como propósito general lograr que la educación que se ofrezca sea intercultural bilingüe, para que así satisfaga con calidad, equidad y pertinencia sus necesidades educativas y básicas de aprendizaje. Es decir, que el niño podrá intercambiar sus conocimientos, creencias, saberes que posee.

\subsection{El Enfoque Intercultural Bilingüe}

En los Artículos $3^{\circ}$ y $4^{\circ}$ constitucionales, la Ley General de Educación y el Programa de Desarrollo Educativo 1995-2000, plantean el compromiso del Estado Mexicano responder a la necesidad de promover en todos los habitantes del territorio nacional, pero sobre todo en las niñas, niños y jóvenes, el desarrollo de las actitudes de tolerancia y respeto a las diferencias que constituya la diversidad cultural.

En el ámbito educativo, se plantea como estrategia educativa para transformar las relaciones entre sociedades, culturas y lenguas de una perspectiva de equidad, calidad y pertinencia, construyendo respuestas educativas diferentes y significativas y como enfoque metodológico para considerar los valores, saberes, conocimientos, lenguas y otras expresiones culturales como recursos para transformar la práctica docente. La educación indígena deberá ser flexible y de respeto promoviendo la valoración de ellas mismas.

La diversidad, se presenta en las dimensiones étnicas, lingüísticas, y cultural respondiendo a dinámicas sociales distintas por lo que cada una de ellas tiene que ser atendidas en el proceso educativo de acuerdo a sus características. Ya que la diversidad cultural es una construcción social históricamente determinada por la que se demanda para satisfacer las necesidades y demandas en el campo de la educación. 
Desde este enfoque la educación bilingüe favorece la adquisición, fortalecimiento desarrollo y consolidación tanto de la lengua indígena y las prácticas cotidianas que lleva en la vida.

La educación intercultural se caracteriza por considerar la diversidad cultural como un recurso para el enriquecimiento y potenciación de la educación de igual manera lleva consigo una formación que se observa a través de actitudes básicas como son:

- $\quad$ El respeto a toda persona

- El respeto a la autonomía de los individuos, de los pueblos y de las culturas

- El apego a la legalidad

- La aceptación interpersonal

- La corresponsabilidad social

- La aceptación positiva de las diversas culturas

- La autoestima

- El respeto a todo pueblo y a su propia cultura

- La tolerancia de ideas y de conductas individuales y grupales que no sean contrarias a los derechos humanos

- La lucha activa contra la discriminación racial

- La superación del egoísmo y de etnocentrismo

- La cooperación activa en la construcción social

- La conservación del medio natural

- La autonomía

- El sentido crítico personal

En el primer ciclo, debido a la importancia de alfabetizar a los niños hablantes de lenguas indígenas en su idioma materno, se prioriza el uso de la lengua indígena como lengua de instrucción y como objeto de estudio. Por tal razón, durante este ciclo escolar, el $67 \%$ del tiempo asignado al área del lenguaje se utilizará para impartir la asignatura Lengua Indígena, y el $33 \%$ restante para la enseñanza del español como segunda lengua. En el tiempo asignado 
para las otras áreas de estudio se prioriza el uso de la lengua indígena como lengua de instrucción (Lengua Indígena Parámetros Curriculares 2012).

Para el caso de la asignatura estatal: lengua adicional es necesario señalar que su propósito es fomentar que los alumnos cursen desde el último grado de preescolar hasta sexto de primaria la enseñanza de una lengua adicional, la cual puede ser la lengua materna, una lengua extranjera o el español como segunda lengua, para el caso de los alumnos que tengan una lengua indígena como lengua materna. Los contenidos de esta asignatura serán seleccionados y diseñados por cada entidad, a partir de los lineamientos nacionales y de acuerdo con las características, las necesidades y los intereses de sus alumnos (Plan de estudios, 2011 p. 19).

\subsection{La educación basada en competencias}

La educación, tiene la demanda de dotar a los niños y jóvenes de elementos necesarios que favorezcan sus capacidades y potencialidades, en este sentido la UNESCO ha destacado que la educación debe ser integral con el propósito de cubrir todos los aspectos de la vida con conocimientos científicos (aprender a conocer), destrezas profesionales (aprender a hacer), valores humanos y principios (aprender a ser), y el ejercicio de la responsabilidad ciudadana (aprender a convivir); el enfoque por competencias es un enfoque holístico de la educación, que barca la puesta en práctica conjunta e interrelacionada de conocimientos, habilidades, actitudes y valores para la resolución de problemas específicos de la vida personal, pública y laboral (Secretaría de Educación Pública 2011); a través de este enfoque de competencias para la vida, se busca un desarrollo pleno e integral de los niños.

Este enfoque implica crear situaciones de aprendizaje que impliquen desafíos que les permitan avanzar paulatinamente en sus niveles de logro para aprender más de lo que saben acerca del mundo y para que sean personas cada vez más seguras, autónomas, creativas y participativas; Tobón (2009) define que "las competencias son procesos complejos de desempeño e idoneidad en un determinado contexto, con responsabilidad" (Educación básica basada en competencias p. 5), en otras palabras la manifestación de una competencia revela la puesta en práctica de conocimientos, habilidades, actitudes y valores para el logro de propósitos o 
resolución de problemas en contextos y situaciones diversas, por esta razón se utiliza el concepto de movilización de conocimientos o saberes, que se manifiesta tanto en situaciones comunes como complejas de la vida diaria y ayuda a visualizar un problema, poner en práctica los conocimientos pertinentes para resolverlo, reestructurarlos en función de la situación, así como extrapolar o prever lo que hace falta (SEP, Plan de estudios 2011); bajo este enfoque se propone desarrollar competencias para experimentar una nueva forma de trabajar contenidos, vinculados a problemas reales, con la finalidad de idear estrategias didácticas que permitan un cambio en nuestras prácticas pedagógicas cotidianas; ya que la formación por competencias no sólo significa cambiar la presentación de la información, ni de modificar los materiales de apoyo, sino implica lograr que trabajemos de otra manera con los alumnos.

Las tendencias educativas internacionales apuntan en general a impulsar reformas que mejoren la calidad educativa, la Reforma Integral de Educación Básica (RIEB) 2011 recupera la perspectiva del trabajo bajo un enfoque por competencias que vislumbra la conformación en México de una sociedad de conocimiento plural, democrática y socialmente justa, lo que nos compromete a emprender un conjunto de acciones que debemos asumir si deseamos que los avances de la ciencias, las tecnologías y las humanidades redunden en beneficios para toda la ciudadanía, elevando en índice de competitividad nacional, pero sin detrimento de la calidad de vida de las personas.

\subsection{El campo de la lengua}

Debido a que México es un país pluricultural, se ha buscado la manera de mejorar la educación para las niñas y los niños indígenas respetando la diversidad cultural que existe en cada pueblo, región y estado. Ante esta situación, el Plan y Programa 2011 de la Secretaría de Educación Pública retoma el enfoque comunicativo y funcional del plan y programa de español, cuyo objetivo el alumno de a conocer de manera escrita y oral su experiencia misma que ellos también podrán intercambiar sus saberes para mejorar y desarrollar las competencias

y componentes educativas. El componente que se le da prioridad en la propuesta pedagógica 
es la escritura que pretende que los niños logren un dominio paulatino de la producción de textos, pero en la lengua indígena.

Los contenidos de este componente se organizan entre apartados:

Conocimiento de la lengua escrita y otros códigos gráficos. El propósito es que los niños utilicen las características del sistema de escritura.

Funciones de la escritura, tipo de texto y características. Este propicia que los niños conozcan e incluyan en sus escritos las características de forma y contenido del lenguaje, propias de diversos tipos de texto, de acuerdo con los propósitos que desean satisfacer.

Producción de textos. El propósito es que los niños conozcan y utilicen estrategias para organizar, redactar, revisar y corregir la escritura de textos de distinto tipo y nivel de complejidad.

Pero para poder lograr esta competencia se hace uso del componente de la expresión oral que igual manera consiste en mejorar paulatinamente la comunicación oral de los niños interactuando situaciones y experiencias vividas en casa con la familia y escuela.

Para el mejoramiento de la expresión oral los contenidos se han organizado en tres aspectos:

Interacción en la comunicación. El propósito es que el niño logre escuchar y producir en forma comprensiva los mensajes, considerando los elementos que interactúan en la comunicación y que pueden condicionar el significado

Funciones de la comunicación oral. El propósito es favorecer el desarrollo de la expresión verbal utilizando el lenguaje para dar y obtener información, conseguir que otros hagan algo, planear acciones propias 
Discursos orales, intenciones y situaciones comunicativas. Se propone que el alumno participe en la producción y escucha compresiva de distintos tipos de discurso, advirtiendo la escritura de estos y considerando el lenguaje, según las diversas intenciones y situaciones comunicativas

\section{Materiales y Método}

La perspectiva metodológica que se ha utilizado ha sido cualitativa. Se pretende comprender la experiencia y los factores que inciden en un fenómeno educativo, considerando la concepción de los individuos (Hernández-Sampieri, 2016). El grupo de informantes estuvo constituido por 12 profesores de educación primaria indígena y sus alumnos de la escuela primaria bilingüe Francisco I. Madero, de la localidad de Huitexcalco pertenecientes a la zona 055 del municipio de Chilcuahutla. Uno de los requisitos para participantes fue que estuvieran asistiendo al curso titulado Lengua Indígena Mexicana Hñahñu, así como disposición para realizar la entrevista y cruzar información, la cual permitió valorar el nivel de alfabetización bilingüe al generar aprendizajes en la lectoescritura en alumnos.

La recogida de la información se hizo mediante cuestionario con preguntas semiestructuradas, para lo cual se desarrolló un guion abierto, planteando cuestiones en las que se encontraban reflejados los temas y subtemas que debían cubrirse sobre la alfabetización bilingüe a docentes. Después de cada entrevista individual, resultó conveniente tomar notas de campo sobre el docente entrevistado, su comunicación no verbal, etc., lo que fue de gran ayuda al momento de redactar los datos de las entrevistas.

Por otra parte, se realizó la misma acción con el grupo de alumnos, lo que permitió valorar el grado de interés y el avance en la asignatura de Hñahñu. Atendiendo a las características de la investigación cualitativa, el análisis de los datos se llevó a cabo de modo reflexivo durante todo el proceso de la investigación, acompañado de una serie de imágenes y videos. De cada entrevista se extrajo el enunciado más representativo para agruparlo en una tabla. Cada una 
de las descripciones se consideró en la interpretación. De esta manera, se obtuvo una descripción detallada de las experiencias y significados adquiridos por los informantes.

Con el fin evaluar la calidad de los datos y los resultados del estudio se siguió el procedimiento propuesto por Hernández (2016), el cual propone criterios para determinar la fiabilidad de los datos cualitativos:

Confiabilidad, se refiere a si el investigador ha captado el significado completo y profundo de las experiencias de los participantes particularmente de aquellos vinculados con el planteamiento del problema. La pregunta a responder es ihe recogido, comprendido, y transmitido en profundidad y con amplitud de significado, vivencias y conceptos de los participantes?

Transferibilidad, se refiere a la posibilidad de transpolar los resultados a otros ámbitos, incluso que parte de ellos o su esencia puedan aplicarse en otros contextos, dando pauta para que los estudios cualitativos produzcan datos de gran valor social al tener una idea general del problema estudiado y la posibilidad de aplicar ciertas soluciones en otro ambiente.

Confirmación, este criterio está vinculado a la credibilidad y se refiere a demostrar que se ha minimizado los sesgos y tendencias del investigador, implica rastrear los datos en su fuente y la explicitación de la lógica utilizada para interpretarlos.

\section{Descripción de los resultados}

Con la finalidad de aprovechar el discurso de las narrativas obtenidas durante la recogida de datos, he ordenado la exposición de los hallazgos según las preguntas y observaciones propuestas para el análisis en consecuencia arrojo los resultados más relevantes para la investigación. Todo con el fin de que la idea propuesta sea captada de la mejor manera posible. Es importante mencionar que los resultados de la presente investigación, al tratarse de valoraciones subjetivas realizadas por los participantes y narradas retrospectivamente, acerca 
de sus experiencias relacionales y comunicativas vividas en un momento de su vida profesional, no pueden considerarse como, el significado final de dicha experiencia, ya que ésta cambia conforme la persona madura incluso mejoran a lo largo de su vida profesional.

\subsection{Categorías de los docentes}

\begin{tabular}{|c|c|c|}
\hline RESPUESTAS & PREGUNTAS & INTERPRETACIONES \\
\hline $\begin{array}{l}\text { - Parte del legado } \\
\text { - Identidad } \\
\text { - Contexto } \\
\text { - Origen } \\
\text { - Valor cultural. } \\
\text { - Conservación } \\
\text { - Legado. } \\
\text { - Lengua necesaria. } \\
\text { - Lengua útil. } \\
\text { - Idioma bonito. } \\
\text { - Diversidad. }\end{array}$ & $\begin{array}{l}\text { 1. ¿Qué significa para ti la } \\
\text { lengua indígena Hñahñu? }\end{array}$ & $\begin{array}{l}\text { - La lengua hñähñu tuene gran } \\
\text { importancia para los docentes pues de } \\
\text { acuerdo a las respuestas, da identidad, } \\
\text { es parte de cultura de nuestros } \\
\text { antepasados, así como parte de la } \\
\text { diversidad no obstante para algunos } \\
\text { es solo una lengua necesaria, útil y } \\
\text { bonita. }\end{array}$ \\
\hline $\begin{array}{l}\text { - Rescatar la lengua en el } \\
\text { ámbito oral y escrito. } \\
\text { - Aprender la lengua } \\
\text { - Aprender a hablar } \\
\text { - Aprender a escribirlo. } \\
\text { - Realizar traducciones. } \\
\text { - Transmitir a los alumnos } \\
\text { - Poder comunicarme con } \\
\text { hablantes Hñahñu } \\
\text { - Sentirme orgullosa de } \\
\text { dominar la lengua. } \\
\text { - Conservar el origen. } \\
\text { - Perfeccionar la lengua. }\end{array}$ & $\begin{array}{l}\text { 2. ¿Con que? propósito } \\
\text { asistes al curso titulado } \\
\text { ‘lengua indígena mexicana } \\
\text { Hñahñu? }\end{array}$ & $\begin{array}{l}\text { Esta respuesta es igual de importante } \\
\text { ya que al observar encuentro, la } \\
\text { necesidad de aprender esta lengua } \\
\text { principalmente porque los que } \\
\text { asisten al curso son monolingües en } \\
\text { español, así mismo tienen la } \\
\text { necesidad y responsabilidad de } \\
\text { conservar la lengua como dominarla. }\end{array}$ \\
\hline $\begin{array}{l}\text { - Respeto y valor a las } \\
\text { personas bilingües } \\
\text { - Cambios interculturales. } \\
\text { - En lo personal. }\end{array}$ & $\begin{array}{l}\text { 3. ¿Describe en que aspectos } \\
\text { te ha generado cambios? }\end{array}$ & $\begin{array}{l}\text { - La pregunta pide los cambios que te } \\
\text { ha generado y en su mayoría, los } \\
\text { profesores respondieron que personal } \\
\text { y hacen referencia a estos cambios, }\end{array}$ \\
\hline
\end{tabular}




\begin{tabular}{|c|c|c|}
\hline $\begin{array}{l}\text { - Laboral y/o profesional. } \\
\text { - Vocabulario amplio } \\
\text { - Reconocimiento de las reglas } \\
\text { ortográficas } \\
\text { - Mejorar pronunciación. } \\
\text { - Aprender su estructura } \\
\text { gramatical. } \\
\text { - Nuevas estrategias y } \\
\text { aprendizajes. } \\
\text { - Valor de la identidad. } \\
\text { - Seguridad en trabajo } \\
\text { docente. }\end{array}$ & & $\begin{array}{l}\text { es un tanto sorprendente porque solo } \\
\text { tres personas mencionaron cambios } \\
\text { en el aspecto laborar. }\end{array}$ \\
\hline $\begin{array}{l}\text { - Aplicar lo aprendido } \\
\text { - Comunicación en la lengua } \\
\text { Hñahñu. } \\
\text { - Aprendizaje al hablarlo y } \\
\text { escribirlo } \\
\text { - Las dinámicas. } \\
\text { - La interacción. } \\
\text { - En ambiente de trabajo } \\
\text { - Hacer escritos cortos. }\end{array}$ & $\begin{array}{l}\text { 4. Durante el curso ¿Cuál ha } \\
\text { sido la mejor experiencia } \\
\text { educativa? ¿Por qué? }\end{array}$ & $\begin{array}{l}\text { •El asistir al curso realmente ha } \\
\text { generado aprendizajes significativos } \\
\text { a partir de experiencias. Pues las } \\
\text { respuestas dan cuenta de sus } \\
\text { conocimientos adquiridos. }\end{array}$ \\
\hline $\begin{array}{l}\text { - Interés en el significado de } \\
\text { palabras } \\
\text { - Interés en el hablarlo } \\
\text { - Interés en escribirlo } \\
\text { - Gusto. } \\
\text { - Empeño. } \\
\text { - Esfuerzo. } \\
\text { - Mucho. } \\
\text { - Sin apoyo de padres. } \\
\text { - La valoran. } \\
\text { - Muy poca por influencia de } \\
\text { los padres. }\end{array}$ & $\begin{array}{l}\text { 5. ¿Qué interés muestra tus } \\
\text { alumnos hacia la lengua } \\
\text { Hñahñu? }\end{array}$ & $\begin{array}{l}\text { - Permite analizar el interés que } \\
\text { observan los profesores hacia sus } \\
\text { alumnos el cual arroja que tienen } \\
\text { mucho interés en aprender o } \\
\text { practicar esta lengua y aun que dos } \\
\text { profesores mencionan que los tutores } \\
\text { son el obstáculo porque no son } \\
\text { hablantes Hñahñu y hay poca } \\
\text { influencia. }\end{array}$ \\
\hline
\end{tabular}




\subsection{Categoría de los alumnos}

\begin{tabular}{|c|c|c|}
\hline RESPUESTAS & PREGUNTAS & INTERPRETACIONES \\
\hline - Viernes $1 \mathrm{hr}$. & $\begin{array}{l}\text { 1. ¿Qué días tienes clases de } \\
\text { Hñahñu? }\end{array}$ & $\begin{array}{l}\text { - De acuerdo a la respuesta es } \\
\text { mínimo el tiempo que se le } \\
\text { dedica a la lengua. }\end{array}$ \\
\hline $\begin{array}{l}\text { - } \text { Poco } \\
\text { - } \text { No se entiende. } \\
\text { - Es difícil. } \\
\text { - Es divertida. } \\
\text { - Más o menos } \\
\text { - Aprender como los abuelos } \\
\text { - } \mathrm{No} \mathrm{se} \\
\text { - } \mathrm{No} \\
\text { - } \mathrm{Si}\end{array}$ & $\begin{array}{l}\text { 2. ¿Te gusta o no hablar la } \\
\text { lengua Hñahñu? } \\
\text { ¿Por qué? }\end{array}$ & $\begin{array}{l}\text { El gusto o no por la lengua, que } \\
\text { tienen los niños realmente fue } \\
\text { muy variado se observa la } \\
\text { necesidad de aprenderlo, pero se } \\
\text { les hace difícil y eso quita el } \\
\text { gusto. }\end{array}$ \\
\hline 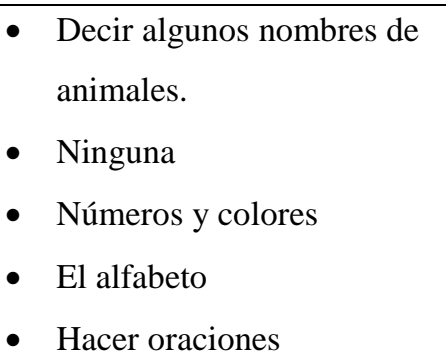 & $\begin{array}{l}\text { 3. ¿Cuál ha sido la mejor } \\
\text { experiencia en la clase de } \\
\text { Hñahñu? }\end{array}$ & $\begin{array}{l}\text { De acuerdo a las respuestas si } \\
\text { son varios los temas que han } \\
\text { abordado y aunque algunos } \\
\text { menos significativos que otros. }\end{array}$ \\
\hline $\begin{array}{l}\text { - } \mathrm{Si} \\
\text { - } \mathrm{No}\end{array}$ & $\begin{array}{l}\text { 4. ¿Te interesa aprender más de } \\
\text { lengua Hñahñu? }\end{array}$ & $\begin{array}{l}\text { - Todos mostraron interés por } \\
\text { aprender. }\end{array}$ \\
\hline $\begin{array}{l}\text { - } \text { Axi } \\
\text { - } \text { Banjua } \\
\text { - Sefi } \\
\text { - Ke'ña } \\
\text { - N’a } \\
\text { - } \text { Oni. } \\
\text { - Imu } \\
\text { - Tsatio }\end{array}$ & $\begin{array}{l}\text { 5. Escribe algunas palabras que } \\
\text { conozcas en Hñahñu }\end{array}$ & $\begin{array}{l}\text { - Anteriormente mencionaron los } \\
\text { temas que les agradaron, pero en } \\
\text { esta pregunta dan a conocer su } \\
\text { aprendizaje logrado y } \\
\text { únicamente escribieron nombres } \\
\text { de animales. }\end{array}$ \\
\hline
\end{tabular}




\section{Conclusiones}

Las consideraciones finales, están escritas a modo de reflexión sobre el fenómeno de estudio, se evidenciaron situaciones que en teoría están planteadas, tales como; que la lengua hñähñu tiene gran importancia para los docentes pues de acuerdo a las respuestas, da identidad, es parte de la cultura ancestral, así como parte de la diversidad; no obstante, para algunos es solo una lengua útil y bonita.

Es importante rescatar la responsabilidad de los docentes por preservar la lengua, que, como ellos mencionaron es un legado de generaciones que se está perdiendo. Además, resulta un tanto complicado preservarla en especial para maestros que son monolingües en español, es

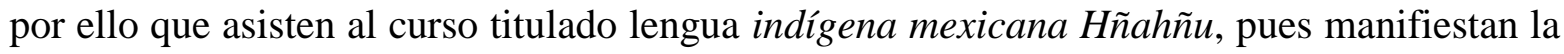
necesidad de aprenderla principalmente, por que tienen la responsabilidad de impartir clases en sus lugares de trabajo y de propiciar aprendizajes significativos de esta lengua retomando el contexto de los niños y sus aprendizajes previos.

La participación de los docentes en el curso de capacitación ha generado aprendizajes significativos a partir de experiencias, pues las respuestas dan cuenta de sus conocimientos adquiridos como; mantener una comunicación en la lengua Hñahñu, el poder hablarlo y escribirlo, aunque sean textos cortos, conocer nuevas dinámicas que pueden ser aplicables en las aulas, se genera ambiente de trabajo que permite interactuar y compartir experiencias.

Los profesores manifiestan de acuerdo a su observación que existe interés de los alumnos por aprender y practicar, así mismo hacen referencia a que los tutores en algunas situaciones son el obstáculo de que se practique la lengua porque no son hablantes Hñahñu y hay poco o nulo seguimiento en casa. Respecto al programa de estudio, se considera poco tiempo que se le destina a esta asignatura y aunque son varios los temas que han abordado, es importante priorizarlos en función a la utilidad y funcionalidad del aprendizaje. 
Otras de las cuestiones fue el gusto por aprender la lengua, se observa interés de aprenderlo a pesar de manifestar dificultad para hablarlo o escribirlo. Estos resultados permitieron identificar el nivel de alfabetización bilingüe mediante la producción de textos, así como las oportunidades de mejora. En adelante se busca el desarrollo e implementación de estrategias que permitan lograr mayor aprendizaje.

\section{Referencias}

Bonfil, G (1990). El indio reconocido en México profundo, una civilización negada, (1 . Edición) México, D.F.: Grijalbo, Pp. 45-51.

Constitución Política de los Estados Unidos Mexicanos. http://www.diputados.gob.mx/LeyesBiblio/pdf/1_080520.pdf

Dirección General de Educación Indígena DGEI/SEP (2011). La lengua de los mayores en: Lo que nos queda en el corazón. México, D.F.: SEP, pp.121-147.

Dirección General de Educación Indígena. (2008). Lengua Indígena parámetros curriculares. Documento curricular para la elaboración de los programas de estudio de las lenguas indígenas.

Hernández-Sampieri. (2014) Metodología de la investigación: enfoque cualitativo. (6º edición) México D.F: Mc. Graw Hill

Instituto Nacional de Lenguas Indígenas-SEP. Norma de la escritura de la lengua Hñahñu. $1^{\circ}$ edición. México, DF. En: www.inali.gob.mx

Lengua Indígena Parámetros Curriculares. Documento curricular para la elaboración de los programas de estudio de las lenguas indígenas, México, SEP. https://programainfancia.uam.mx/pdf/publicaciones/lengua_indigena/parametros.pdf 
Ley General de Derechos Lingüísticos de los Pueblos Indígenas. Nueva ley publicada en el Diario Oficial de la Federación 18-06-2010.

Secretaria de Educación Pública. (2001). Desarrollo del niño y del adolescente compendio para educadores en la Biblioteca para la Actualización del Maestro. (1 ${ }^{\circ}$ Reimpresión). México, D.F. SEP/McGraw-Hill Interamericana, pp16-40.

Secretaria de Educación Pública. Parámetros curriculares de la asignatura de lengua indígena.

Secretaria de Educación Pública. Plan de Estudios 2011. Educación Básica. https://www.gob.mx/cms/uploads/attachment/file/20177/Plan_de_Estudios_2011_f.pdf

Secretaria de Educación Pública. Reforma Integral de la Educación Básica 2011. Diplomado para docentes de primaria $3^{\circ}$ y $4^{\circ}$ grados. https://zona141 camargotam.files.wordpress.com/2011/11/diplomado-mod-1-3-y-4-20112012.pdf

Secretaria de Educación Pública. Curso Básico de Formación Continua para Maestros en servicio. El enfoque por competencias en la educación básica 2009, p. 5. http://www.sev.gob.mx/virusinfluenza/docentes/curso_basico_formacion.pdf

Sepúlveda, G. (2014). Interculturalidad y construcción del conocimiento. Reflexiones Pedagógicas, p.7.

Tobón, S. (2009). Formación basada en Competencias. Pensamiento complejo, diseño curricular y didáctica. (2 $2^{\mathrm{a}}$ Edición). Bogotá, Colombia Ecoediciones. http://200.7.170.212/portal/images/documentos/formacion_basada_competencias.pdf 